\title{
GarCoSim: A Framework for Automated Memory Management Research and Evaluation
}

\author{
Konstantin Nasartschuk \\ kons.na@unb.ca \\ Md. Mazder Rahman \\ p3sp4@unb.ca
}

\author{
Marcel Dombrowski \\ marcel.dombrowski@unb.ca \\ Kenneth B. Kent \\ ken@unb.ca
}

\author{
Tristan M. Basa \\ tbasa@unb.ca \\ Gerhard W. Dueck \\ gdueck@unb.ca
}

Faculty of Computer Science, University of New Brunswick, Fredericton, Canada

\begin{abstract}
Many modern programming languages rely on memory management environments that are responsible for allocation and deallocation of objects. Garbage collection phases are used in order to detect inaccessible objects on the heap so they can be deallocated. The performance of garbage collection techniques depends heavily on the environment, implementation specific parameters and the benchmark used. The contribution of this publication is an extendable memory management simulator, which aims to assist developers in memory management evaluation and research. The simulator is capable of reading operations from a trace file extracted from a virtual machine and simulating the memory management needed by the simulated mutator. The framework aims to provide an isolated experimentation and comparison platform in the field of automatic memory management. New algorithms can be added to the framework in order to compare them to established algorithms.
\end{abstract}

\section{CCS Concepts}

-Software and its engineering $\rightarrow$ Garbage collection; Allocation / deallocation strategies; $\bullet$ Computing methodologies $\rightarrow$ Modeling and simulation;

\section{Keywords}

garbage collection, memory management simulation, trace file

\section{INTRODUCTION}

As programming languages evolved, memory management went through many developments, including the creation of an abstraction layer to hide the underlying complexity of resource management and software engineering. Automated memory management is one of the key features of many modern programming languages.

Explicit memory allocation, as used in $\mathrm{C}$ or $\mathrm{C}++$, requires developers to manage all objects created on the heap explicitly. Objects have to be allocated when they are created and deallocated once the application no longer references them. Studies suggest that $20 \%$ of the time consumed by a project is used for memory management planning and optimization [11].

Automated memory management systems use garbage collectors that manage all objects allocated on the heap and free them once they become inaccessible. Garbage collection algorithms such as reference counting [5, 11], marksweep [15], and mark-compact [10] have emerged in order to detect unused objects. Most of these techniques are based on a stop-the-world concept. The managed application has to pause execution so the garbage collector can identify and free dead objects. The count and the duration of the stopthe-world phases are two of the main metrics indicating the performance of a garbage collector.

Attempts to optimize these metrics include divided heap structures, concurrency, and generational memory management structures [11]. Research and evaluation of algorithms used in automated memory management are implementation dependent and vary, based on the Virtual Machine (VM) utilized, the application that is being managed by the $\mathrm{VM}$, and user defined parameters involved in the algorithm itself.

Currently, new GC algorithms are evaluated and compared using benchmark suites such as SPECjbb [6], DaCapo [2], SPECjvm [7] and others. This paper discusses an approach of creating an evaluation framework for garbage collection techniques which does not rely on a specific virtual machine. The idea of this project is to provide a memory management simulator, which replaces the VM with a simulation of its memory management capabilities. The application is replaced by a trace file which is extracted from an existing virtual machine.

In addition, the framework is developed in an extendable, open source form, so researchers can evaluate and compare new techniques to the results of others in an isolated environment. The memory management toolkit (MMTK) [1], 
which utilizes the Jikes Research Virtual Machine (Jikes RVM) [14], is written in Java and aims to assist researchers in prototyping virtual environment features in a simple virtual machine rather than removing the compilation of the application (mutator) code and a complete isolation of memory management.

A goal of the presented framework is to be simple. Each virtual machine offers a number of optimizations on a variety of levels, such as garbage collection, allocation, just in time compilation and object modelling. Comparing different techniques having such a vast number of parameters can be quite difficult and the search for the source of benefits or causes for disadvantages can take significant time.

The paper is divided into four sections. Section 2 provides a short introduction of automated memory management. To test the memory management simulator, trace files of real world applications were extracted from a JVM. The experimental setup used for this paper is described in Section 3. The extraction process and resulting trace files are topics of Section 4. The design and development of the memory management simulator that allows developers to implement garbage collection techniques is described in Section 5. Results achieved using the combination of trace files and the simulator are presented and discussed in Section 6 .

\section{BACKGROUND}

As Java and other virtual machine-based languages continue to become more popular, the performance of those languages becomes more important. One major drawback of garbage collected languages is still inefficiency, especially for computationally intensive scientific applications [13].

Four memory management techniques build the core of the standard repertoire of virtual machines: Reference counting, Mark-sweep, Mark-compact, and Copying collection. The techniques, excluding reference counting, are introduced in this section.

\subsection{Mark-sweep and mark-compact}

The mark-sweep garbage collection policy treats the heap structure as a directed graph. Objects are connected using their references to each other. The roots of the graph are represented by pointers in registers, global variables, and variables existing on the stack.

The algorithm is divided into a marking and a sweeping stage. The policy requires a stop-the-world stage - at least for the marking stage [15]. Marking describes the traversal of the object graph and marking all objects reached as alive. Once the traversal is done, the sweeping stage can free the space used by dead objects. One downside of the policy is the stop-the-world phase itself, as the mutator is stopped completely and the time is used for memory management. The main problem of this policy is the fragmentation that occurs when objects are freed.

This drawback can be handled by replacing the sweep stage by a more complex compaction phase. Once all live objects are marked, the objects are compacted to remove all fragmentation from the heap. The space located after the last memory location used is marked as free. Compared to the sweep phase, the compaction requires more time. However, the benefit of not having fragmentation has a large impact on the number of garbage collections required in order to accommodate the mutator. The compaction phase can be initiated for every garbage collection or when the fragmentation becomes too high.

\subsection{Copying collection}

The natural extension of the mark-compact collection is represented by the copying collector $[9,4]$. The idea behind this policy is to remove the marking stage and to only use a compaction phase. In order to accomplish this, live objects have to be copied during traversal. As it is not certain how much space and which addresses will be freed during the process, objects have to be copied into an entirely separate address space. This requires the heap space to be divided into two halves. One, where new objects are allocated and the second space where live objects will be copied during a garbage collection. The benefits are a very short stop-theworld time and the fact that no fragmentation is possible. The largest disadvantage is the fact that the effectively available heap space is half of the physical memory space.

\subsection{Combination of policies}

Mixed policies appeared that use the knowledge of common application behaviour. An example is the knowledge that many objects are small and die after a short time while large and old objects have the tendency to survive even longer. In order to use this knowledge, the generational garbage collection technique was introduced. The idea behind it is to divide the heap into a young and an old space or spaces. The space of allocation is therefore collected more often than the old space. In exchange, the allocation space is usually only a small part of the heap, keeping the stop-theworld times very short. Objects surviving long enough are promoted into the old space as they are expected to survive even longer.

\section{EXPERIMENTAL SETUP}

Measuring improvements in automated memory management (MM) performance requires at least the simulation of basic MM operations of a virtual machine (VM). An online simulation is considered expensive as it requires a copy of the VM and an actual run of the benchmark. An alternative approach is to run the simulation off-line using trace files. Trace files offer the flexibility of being able to develop a tailored simulator that focuses only on the basic MM operations and at the same time, be portable to be compiled and run on different platforms.

Trace files were acquired by instrumenting a Java Virtual Machine (JVM), particularly the memory management module. Instrumentation is done by inserting hooks in the JVM code that handle memory-related operations. For each of the operations, a line containing relevant information is written to a file. This output file is post-processed to conform to the standard format specified in Section 4.2. The post-processed file, or trace file, serves as input to the memory management simulator (MMS). It treats the trace file lines as mutator memory operations, allocates space, manages references and performs garbage collections in order to accommodate the requirements of the application.

\section{JVM INSTRUMENTATION \& TRACE}

The JVM's $\mathrm{C} / \mathrm{C}++$ code was modified to log all basic memory management operations into trace files. In this section the MM operations that are captured at JVM runtime are discussed, followed by post-processing of trace files. 


\subsection{Basic Memory Management Operations}

A real life JVM was instrumented to trace four basic MM operations that are described as follows:

\subsubsection{Allocation}

Most objects allocated by Java applications are short-lived [3]. Given the overhead of allocating an object from the general heap, also known as the slow-path allocation, this becomes a bottle-neck in system performance. To circumvent this, performance-oriented JVMs have provisions for assigning regions of memory for exclusive use of a thread. Objects that are identified as being short-lived are allocated from these exclusive regions, also known as fast-path allocation, thereby avoiding the need for expensive synchronization operations. Within both the slow-path and fast-path allocations, it is distinguished between single and indexable (arrays) objects. With fast-path indexable objects, it is distinguished between contiguous and discontiguous arrays. All in all, five types of allocation are identified. For every allocation, the information traced includes the names of threads handling the object allocation, the object id, the size of the object, the number of object reference slots, the class name of the object, the total instance size of the class, and the number of static fields of the class.

\subsubsection{Store Access}

Four different store accesses are as follows:

1. Store primitives into an object: This operation is performed when a variable of an object field is assigned/changed to/with data of a primitive type. The recorded data are thread name, object id, field size, field offset, and field type (volatile/non-volatile).

2. Store references into an object: This operation happens when an object references another object. Relevant information needed for this operation includes the object IDs of both the parent object and the child object, and the reference slot number. The JVM has implemented a write barrier whenever fields are going to be stored into. Hooks were added in these barriers to output relevant information such as the thread, the parent and the child object, and the object reference slot where the child object will be pointed from.

3. Store primitives into a class: This operation is performed when a static variable of a class field is assigned/changed to/with data of a primitive type. The recorded data are thread name, class name, field size, field offset and field type (volatile/non-volatile).

4. Store references into a class: This operation is similar to object-to-object referencing except that the object reference slot is a static field. The recorded information is thread name, class name, field offset/index, object id, field size and field type (volatile/non-volatile).

\subsubsection{Read Access}

An object is also instrumented whenever it (or one of its fields) is accessed. Information from this operation can be used for analyzing the effects of caching.

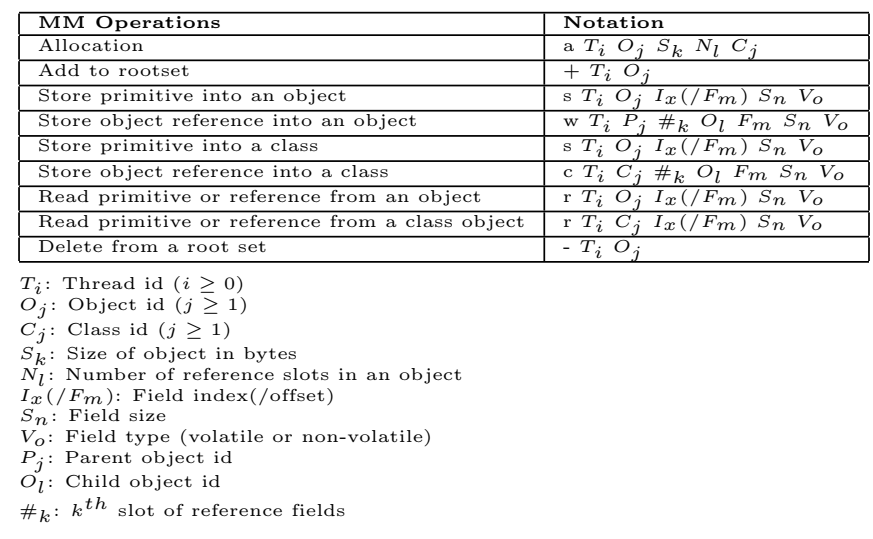

Table 1: The trace file format.

\subsubsection{Rootset Dump}

Rootset dumps are a way of inspecting the thread's rootset. Since this should be done in a stop-the-world fashion, an asynchronous-handler is signaled whenever allocation is done. The timing of the actual dump depends entirely on the JVM. Rootset additions and rootset deletions are inferred between two rootset dumps in the next phase.

\subsection{Post-Processing and Trace File Format}

The instrumented JVM produces a file that contains raw information captured during the execution of an application. Some of this information can be converted to a format that is more simulator-friendly (e.g. conversion of unique identifiers from string to integer). In addition, rootset dumps need to be converted to a series of operations (denoted by ' + ' and '-') in as much as the trace file is interpreted as a sequence of operations.

Another purpose of post-processing is to defer rootset deletions. At some point, some objects become garbage in the trace file but somehow manage to appear again at some later operations. These objects are allocated by the native code and are not captured in the JVM C++ code. Some approaches move them into what is called zombie regions to be resurrected again at some later time [8]. The deletion is deferred from the rootset of its allocating thread until its last access. This prevents it from being collected at least until its last access. Table 1 shows the format of the trace files used in the experiments. Some attributes of classes are captured in class lists. Attributes of a class are denoted by $C_{i} I_{j} \# k$ className, where, $i, j$ and $k$ represent the class id, class object size in bytes, and number of static references.

\subsection{Results of Trace Files}

Experiments are performed with both DaCapo [2] and SPECjvm2008 [7] benchmark suites. When running the instrumented JVM, the Just-in-time (JIT) compiler as well as the packed object feature were disabled. The optthruput GC policy was used to disable concurrent GC, and specify only a maximum of one GC thread to run. The heap size was set to $16 \mathrm{~GB}$. The output is referred to as a raw trace file that contains operations with relevant information about threads, classes as well as root set dumps. Post-processing results in a trace file with the standard format as specified in Section 4.2. Trace files for eight workloads of DaCapo and for 20 workloads of SPECjvm2008 benchmark suite were 


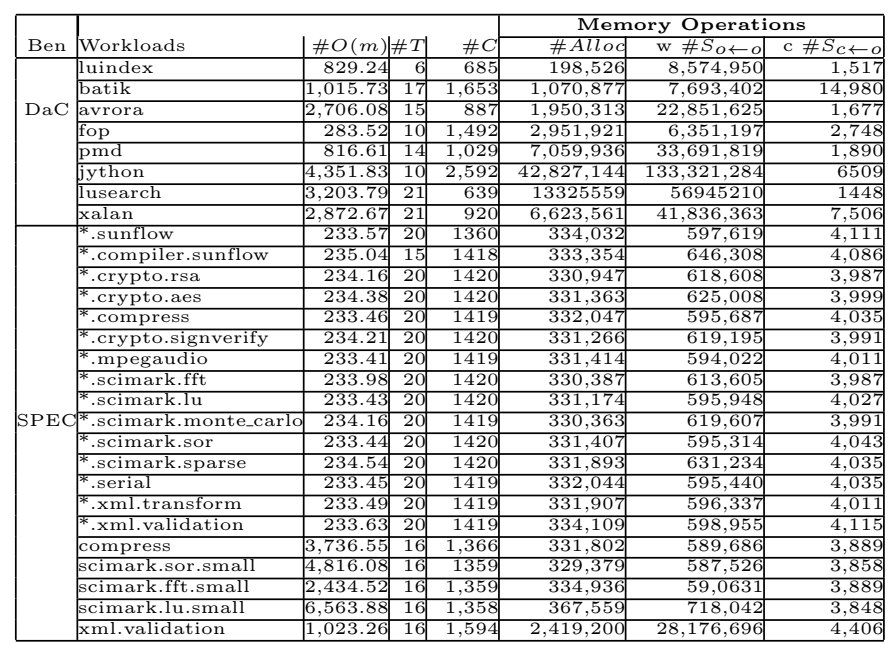

Ben: Benchmark

Dac: DaCapo

SPEC: SPECjvm2008

$\# O(m):$ No. of memory management operations in million

$\# T$ : No. of threads

$\# C$ : No. of classes

\#Alloc: No. of objects allocated

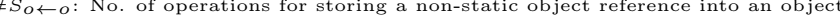

$\# S_{c \leftarrow o}$ : No. of operations for writing a static object reference into a class

Table 2: Metrics of the generated trace files.

generated. Metrics of the generated trace files are shown in Table 2. Column 3 shows the total number of memory management operations captured in each workload running in the JVM. The number of operations that are most relevant to the GC simulator, such as allocations, stores of object references into other objects and stores of object references into class objects are shown in column 6,7 and 8 respectively. Empirical results show that $98 \%$ of all operations are read access operations that can be used in further research to simulate cache misses, object cache locality, etc.

\section{MEMORY MANAGEMENT SIMULATOR}

In order to isolate the memory management aspect of a virtual machine from the mutator execution for evaluation purposes, part of this project was the development of a memory management simulator. The input of the simulator is a trace file containing object allocations and reference operations.

The memory management simulator, developed in $\mathrm{C}++$, utilizes an object-oriented and iteration-based approach. The goal of this development process was to ensure a high extensibility of the framework. At the core of the model are classes representing objects, a memory manager, a garbage collector and the allocator. Even though the memory management simulator was created with a monolithic heap structure in mind, the structure allows developers to create divided heap policies. The framework, including the simulator, the trace generator and their source code are publicly available on GitHub [12].

\subsection{Simulator Structure}

Figure 1 shows the basic class structure of the memory management simulator. The MemoryManager uses the Allocator module for object allocation and keeps track of all existing objects in order to create and maintain references between

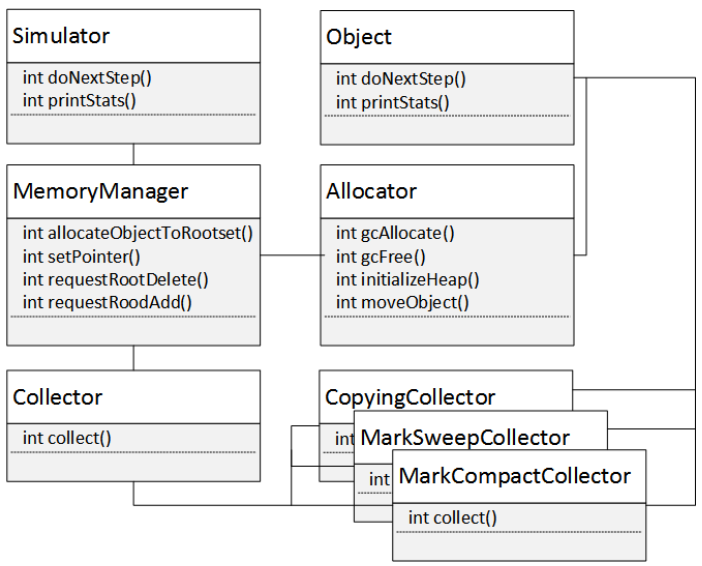

Figure 1: Garbage Collector architecture consisting of MemoryManager, Allocator, Collectors.

them. If an allocation fails or another metric signals that a garbage collection should be initiated, the MemoryManager commands the Collector to do so. The specific implementation of the Collector determines which objects are not referenced any longer and requests a free operation by the allocator.

Only the MemoryManager class can allocate and only the Collector deallocate objects. The mutator can never request the deletion of an object and the garbage collector can never allocate a new object. The only exception to this is the moving process of existing objects during the copying or compacting stage of different collection policies.

A dynamic object container keeps track of all objects that exist on the heap. This includes active and used objects as well as dead objects, which are not yet freed. The root set is used to initiate an object traversal algorithm to locate all live objects. The container can be iterated afterwards in order to find dead objects and to free them, depending on the collection policy. Alternatively, collections and heap organization can be performed without the use of an object list but rather by iterating over the objects on the heap itself using a base pointer and object sizes.

The classes Allocator and Collector are interfaces which can be specified using one of the specific policy implementations developed for it. Allocator implementations include first fit and next fit allocation. GC policies available are mark-sweep, mark-compact, and copying collection.

Additional Allocator and Collector classes can be introduced by implementing the interface calls defined by the parent classes. Examples for both cases are included in the repository. If a changed heap structure is being investigated, changes to the MemoryManager class might be necessary.

The flow of the program starts with the Simulator class, which is responsible for simulation control and input handling. Its main task is to read in memory instructions from the trace file and to advice the MemoryManager which action is currently requested by the mutator. The MemoryManager class represents the orchestration module of the application and routes requests to instances of Allocator or Collector classes depending on metrics, trace file request and the success or failure of allocations. The Object class includes metrics and information about one specific object on the heap. 
It includes the address, object id, creation time and other values, which could be of interest when researching automatic memory management. Additional information can be added if needed.

\section{EXPERIMENTAL RESULTS}

In order to evaluate the simulator, ten trace files extracted from the JVM were processed using the collection policies included in the current version of the simulator. The main metrics for the evaluation of the simulator were the count of garbage collections, the average collection time, and the execution time of the full benchmarks.

As each benchmark has an individual heap structure, the heap size for each run of the benchmarks was increased in $25 \mathrm{MB}$ steps and the change in execution time was recorded. A watermark metric, which triggers a GC before the heap is filled, was not used in any of the tests in order to stress the allocator and garbage collection policies. The machine used for the benchmarks utilized an Intel Core i7-2600 processor and had 8 GB of RAM.

Table 3 shows the total execution time for each benchmark. All combinations of heap size and collection policy are listed. It can be observed that in general, the markcompact policy requires the smallest heap as it does not suffer from fragmentation and does not divide the heap into two separate parts. The copying collection requires the second largest heap and almost always, mark-sweep requires the largest heap in order to be able to run. An extreme case for this metric was the pmd benchmark, which executed successfully using a $100 \mathrm{MB}$ heap when using mark-compact or the copying policy, but required a $250 \mathrm{MB}$ heap in order to finish utilizing mark-sweep garbage collection.

Benchmarks such as compress show the cost of garbage collection. While stop-the-world phases are required, the execution time of the simulation is 25 seconds slower compared to a heap which never becomes full during run time. In addition, this particular case shows once again, that this point is reached with a much larger heap when the copying policy is used.

As described in literature, copying collection was observed to deliver the fastest execution results due to small average collection times [11]. Mark-sweep (when a run finished successfully) had the second fastest results. The compaction phase of the mark-compact policy slowed down garbage collections.

The number of garbage collections for each policy were collected using a $100 \mathrm{MB}$ heap as the heap size allowed for all but two tests to finish while the heap was still small enough to require stop-the-world phases. The last column in Table 3 lists the average garbage collection time for each benchmark when using a heap size of 100MB. The average collection count is listed in parentheses. Those two metrics can be used in order to determine the raw garbage collection time of the benchmark.

An example of garbage collection times and their comparison is the scimark. lu.small benchmark. Even though the collection policy required two collections in order to accommodate the memory needs of the benchmark, the stop-theworld phases were only about 3 seconds on average. The mark-sweep policy had to halt the application for $9.6 \mathrm{sec}$ onds and the mark-compact phase due to its compaction phase 12.2 seconds.

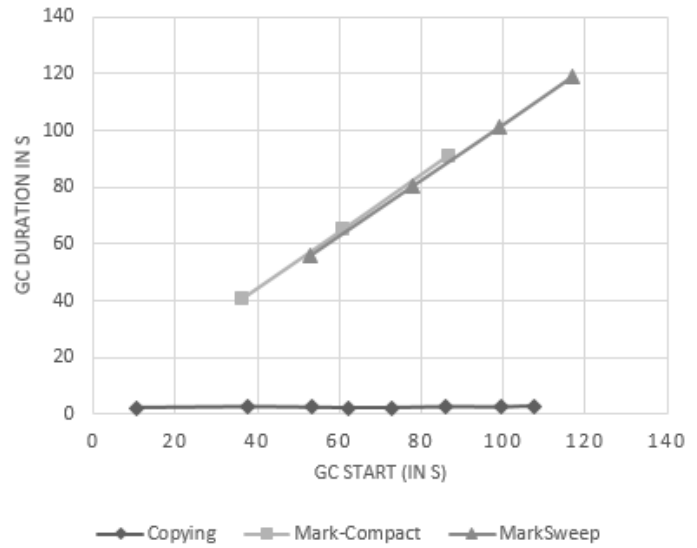

Figure 2: GC start during runtime and its duration when running xml.validation with a heap of $75 \mathrm{MB}$.

The simulator presented is capable of not only comparing different runs using different parameters, but can also be used in order to investigate a single simulation process in more detail. An example would be the investigation of GCs when using the same heap size. In particular the time when a GC happens and how long it takes during the execution process. Using the result log files of the simulator, it is possible to retrieve this data and visualize the information as shown in Figure 2.

The results Figure 2 are as expected. As the copying collector splits the heap in two, the active heap part fills up quickly and collections are required earlier compared to other techniques. On the flipside, the time needed for each collection is constant no matter how many live objects remain on the heap. This is not the case when looking at the graphs of the mark-sweep and mark-compact policies. As the number of live objects and therefore the heap size usually increases, the stop-the-world times of mark-sweep and mark-compact increase as well. This underlines the statement, that a frequently filled heap usually benefits from a copying collection [11].

\section{CONCLUSIONS AND FUTURE WORK}

This paper presented the design and evaluation of a garbage collection simulator which aims to be used for automated memory management research. The structure of the simulator and the process of extracting real application input files for the framework from a JVM was described in detail and the execution and results gathered were discussed in order to show possible applications for the framework.

The simulator is under heavy development. Future work in this project include the development of a trace file generator. It can be used to mimic the memory requirement behaviour of real life applications and to create trace files of any size without the need of extracting them from a virtual machine.

Another field of research is the implementation of additional garbage collection techniques. This includes split heap structures such as generational garbage collection, thread local garbage collection, and others. 


\begin{tabular}{|c|c|c|c|c|c|c|c|c|c|c|c|}
\hline Benchmark & Policy & $50 \mathrm{M}$ & $75 \mathrm{M}$ & $100 \mathrm{M}$ & $125 \mathrm{M}$ & $150 \mathrm{M}$ & $175 \mathrm{M}$ & $200 \mathrm{M}$ & $225 \mathrm{M}$ & $250 \mathrm{M}$ & $\begin{array}{c}\text { Avg GC } \\
\text { time (count), } \\
100 \mathrm{M}\end{array}$ \\
\hline \multirow{3}{*}{ avrora } & $\mathrm{C}$ & 121.28 & 114.193 & 126.047 & 120.397 & 110.032 & 104.892 & 103.865 & 78.715 & 74.651 & $12.466(2)$ \\
\hline & MC & 228.448 & 161.843 & 175.648 & 86.414 & 78.216 & 75.481 & 80.642 & 81.417 & 89.291 & $86.082(1)$ \\
\hline & MS & & & 278.804 & 75.462 & 77.671 & 78.51 & 79.369 & 78.688 & 73.21 & $91.274(2)$ \\
\hline \multirow{3}{*}{ batik } & 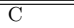 & & 87.864 & $\begin{array}{l}74.575 \\
\end{array}$ & $\overline{772.282}$ & ב64.501 & $\begin{array}{l}58.467 \\
\end{array}$ & 年9.14 & $\begin{array}{ll}60.998 \\
\end{array}$ & $\begin{array}{l}59.906 \\
\end{array}$ & \multirow{3}{*}{$\begin{array}{r}7.751(3) \\
37.363(1)\end{array}$} \\
\hline & MC & 164.921 & 136.528 & 80.491 & 100.637 & 41.526 & 33.3 & 33.425 & 34.083 & 36.259 & \\
\hline & MS & & & & 70.516 & 38.794 & 33.575 & 35.104 & 33.486 & 34.582 & \\
\hline \multirow{3}{*}{ compress } & $\overline{\mathrm{C} \text { C }}$ & & 32.752 & $\begin{array}{l}28.223 \\
\end{array}$ & 22.838 & $\begin{array}{l}24.648 \\
\end{array}$ & 26.661 & $\begin{array}{l}10.266 \\
\end{array}$ & $\bar{~} 10.748$ & 10.921 & 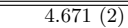 \\
\hline & MC & 46.14 & 35.093 & 9.193 & 9.331 & 9.795 & 9.942 & 9.847 & 10.625 & 11.045 & $0(0)$ \\
\hline & MS & 32.199 & 23.594 & 8.943 & 8.426 & 9.765 & 10.14 & 10.262 & 9.801 & 10.916 & $0(0)$ \\
\hline \multirow{3}{*}{ fop } & $\overline{\mathrm{C}}$ & $\begin{array}{ll}116.718 \\
\end{array}$ & 102.432 & 98.09 & 82.571 & 80.282 & 87.989 & 88.514 & 80.876 & 82.417 & \multirow{3}{*}{$\begin{array}{r}6.524(4) \\
52.806(2) \\
43.985(2)\end{array}$} \\
\hline & MC & 245.933 & 225.998 & 171.13 & 93.13 & 110.713 & 117.605 & 127.162 & 45.862 & 45.412 & \\
\hline & MS & 351.722 & 178.896 & 143.116 & 93.408 & 93.838 & 100.419 & 112.677 & 45.674 & 45.949 & \\
\hline \multirow{3}{*}{ luindex } & $\overline{\mathrm{C}}$ & 30.358 & $\begin{array}{l}25.596 \\
\end{array}$ & 23.609 & 26.655 & $\begin{array}{l}26.076 \\
\end{array}$ & 28.095 & ב26.549 & 21.981 & 29.355 & \multirow{3}{*}{$\begin{array}{l}0(0) \\
0(0) \\
0(0)\end{array}$} \\
\hline & $\mathrm{MC}$ & 22.848 & 27.86 & 27.248 & 26.615 & 27.029 & 26.912 & 26.421 & 27.676 & 28.042 & \\
\hline & MS & 28.29 & 26.515 & 20.535 & 26.243 & 27.041 & 27.272 & 35.583 & 26.566 & 27.578 & \\
\hline \multirow{3}{*}{ pmd } & 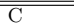 & & 年 408.8 & $\begin{array}{l}292.74 \\
\end{array}$ & $\begin{array}{c}334.743 \\
\end{array}$ & $\begin{array}{l}314.149 \\
\end{array}$ & 291.701 & 239.141 & 209.069 & 216.057 & \multirow{3}{*}{$\begin{array}{r}4.975(17) \\
107.926(6)\end{array}$} \\
\hline & $\mathrm{MC}$ & & & 818.403 & 718.756 & 735.743 & 574.013 & 341.939 & 368.925 & 392.716 & \\
\hline & MS & & & & & & & & & 343.782 & \\
\hline \multirow{3}{*}{ scimark.fft.small } & 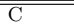 & $\begin{array}{l}27.707 \\
\end{array}$ & $\begin{array}{l}23.477 \\
\end{array}$ & (19.78 & 21.572 & $\begin{array}{l}23.541 \\
\end{array}$ & 25.303 & $\begin{array}{l}10.131 \\
\end{array}$ & $\begin{array}{l}10.487 \\
\end{array}$ & $\begin{array}{l}10.768 \\
\end{array}$ & 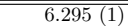 \\
\hline & $\mathrm{MC}$ & 22.656 & 28.955 & 9.147 & 9.232 & 9.531 & 9.807 & 10.18 & 10.454 & 10.831 & $0(0)$ \\
\hline & MS & 19.187 & 23.735 & 9.119 & 9.182 & 9.458 & 10.002 & 10.125 & 10.432 & 10.803 & $0(0)$ \\
\hline \multirow{3}{*}{ scimark.lu.small } & 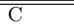 & 24.434 & $\begin{array}{l}22.062 \\
\end{array}$ & $\begin{array}{l}20.075 \\
\end{array}$ & $\begin{array}{l}18.661 \\
\end{array}$ & $\begin{array}{l}19.149 \\
\end{array}$ & $\begin{array}{l}19.416 \\
\end{array}$ & $\begin{array}{l}20.676 \\
\end{array}$ & $\begin{array}{l}22.302 \\
\end{array}$ & 8.623 & 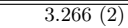 \\
\hline & $\mathrm{MC}$ & 32.042 & 20.913 & 25.298 & 11.349 & 8.095 & 7.941 & 8.483 & 8.59 & 8.623 & $12.2(1)$ \\
\hline & MS & 26.038 & 18.606 & 20.08 & 7.154 & 10.422 & 8.236 & 8.323 & 9.402 & 8.716 & $9.599(1)$ \\
\hline \multirow{3}{*}{ scimark.sor.small } & C & 11.753 & 10.391 & 11.374 & 5.124 & 5.413 & 25.518 & 5.782 & 6.004 & 6.324 & $3.286(1)$ \\
\hline & MC & 15.061 & 4.657 & 4.973 & 5.308 & 5.376 & 5.559 & 5.823 & 6.06 & 6.468 & $0(0)$ \\
\hline & MS & 12.125 & 4.951 & 4.948 & 5.386 & 5.364 & 5.589 & 5.753 & 6 & 6.184 & $0(0)$ \\
\hline \multirow{3}{*}{ xml.validation } & 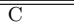 & $\begin{array}{l}180.149 \\
\end{array}$ & 135.206 & $\begin{array}{l}132.794 \\
\end{array}$ & "158.282 & 169.955 & "161.932 & "127.563 & 153.944 & "139.763 & $\begin{array}{c}3.643(6) \\
\end{array}$ \\
\hline & $\mathrm{MC}$ & 735.03 & 330.131 & 273.436 & 338.09 & 227.003 & 280.423 & 203.744 & 193.258 & 199.035 & $83.621(2)$ \\
\hline & MS & & 502.177 & 325.567 & 315.409 & 223.821 & 222.375 & 221.177 & 179.534 & 186.048 & 76.743 (3) \\
\hline
\end{tabular}

Table 3: Execution time of the simulation in seconds. Each benchmark was run with different heap sizes and different collection policies (Copying, Mark-Compact, and Mark-Sweep). An empty cell stands for an insufficiently large heap. The last column lists average GC times when using a heap size of $100 \mathrm{MB}$. The times are listed in seconds.

\section{ACKNOWLEDGMENTS}

The authors would like to acknowledge the funding support provided by the Atlantic Canada Opportunities Agency (ACOA) through the Atlantic Innovation Fund (AIF) program. Furthermore, we would also like to thank the New Brunswick Innovation Fund for contributing to this project. Finally, we would like to thank the Centre for Advanced Studies-Atlantic for access to the resources for conducting our research.

\section{REFERENCES}

[1] S. Blackburn, R. Garner, and D. Frampton. Mmtk: The memory management toolkit, 2006.

[2] S. M. Blackburn, R. Garner, C. Hoffmann, A. M. Khang, K. S. McKinley, R. Bentzur, A. Diwan, D. Feinberg, D. Frampton, S. Z. Guyer, et al. The dacapo benchmarks: Java benchmarking development and analysis. In ACM Sigplan Notices, volume 41, pages 169-190. ACM, 2006.

[3] S. M. Blackburn, R. Jones, K. S. McKinley, and J. E. B. Moss. Beltway: Getting around garbage collection gridlock. In L. J. Hendren, editor, Proceedings of PLDI'02 Programming Language Design and Implementation, pages 182-196, Berlin, June 2002. ACM Press.

[4] C. J. Cheney. A nonrecursive list compacting algorithm. Communications of the ACM, 13(11):677-678, 1970.

[5] G. E. Collins. A method for overlapping and erasure of lists. Communications of the ACM, 3(12):655-657, 1960.
[6] S. P. E. Corporation. SPECjbb2013. https://www.spec.org/jbb2013/ [Online. Last accessed: 2015-06-08], 2015.

[7] S. P. E. Corporation. SPECjvm2008. https://www.spec.org/jvm2008/ [Online. Last accessed: 2015-06-08], 2015.

[8] S. Dieckmann and U. Hoelzle. A study of the allocation behavior of the specjvm98 java benchmarks. Technical report, Santa Barbara, CA, USA, 1998.

[9] R. R. Fenichel and J. C. Yochelson. A lisp garbage-collector for virtual-memory computer systems. Communications of the ACM, 12(11):611-612, 1969.

[10] C. H. Flood, D. Detlefs, N. Shavit, and X. Zhang. Parallel garbage collection for shared memory multiprocessors. In Java Virtual Machine Research and Technology Symposium, 2001.

[11] R. Jones, A. Hosking, and E. Moss. The Garbage Collection Handbook: The Art of Automatic Memory Management. Chapman \& Hall/CRC, 2011.

[12] K. B. K. K. Nasartschuk, M. Dombrowski and G. Dueck. GarCoSim repository website. https://github.com/GarCoSim [Online: last accessed: 2015-06-08], 2015.

[13] J. Mathew, P. D. Coddington, and K. A. Hawick. Analysis and development of java grande benchmarks. In Proceedings of the ACM 1999 conference on Java Grande, pages 72-80. ACM, 1999.

[14] J. RVM. Jikes RVM Home. http://www.jikesrvm.org [Online. Last accessed: 2015-06-08], 2015.

[15] R. A. Saunders. The lisp system for the q-32 computer. The Programming Language LISP: Its Operation and Applications, pages 220-231, 1974. 